\title{
A universal minimal mass scale for present-day central black holes
}

\author{
Tal Alexander*and Ben Bar-Or ${ }^{\dagger}$
}

Intermediate-mass black holes (IMBHs) of mass $M_{\bullet} \approx 10^{2}-10^{5}$ solar masses, $M_{\odot}$, are the long-sought missing link ${ }^{1]}$ between stellar black holes, born of supernovac ${ }^{2}$, and massive black holes ${ }^{3}$, tied to galaxy evolution by the empirical $M_{\bullet} / \sigma_{\star}$ correlation $^{415}$. We show that low-mass black hole seeds that accrete stars from locally dense environments in galaxies following a universal $M_{\bullet} / \sigma_{\star}$ relation ${ }^{6 / 77}$ grow over the age of the Universe to be above $\mathcal{M}_{0} \approx 3 \times 10^{5} M_{\odot}(5 \%$ lower limit), independent of the unknown seed masses and formation processes. The mass $\mathcal{M}_{0}$ depends weakly on the uncertain formation redshift, and sets a universal minimal mass scale for present-day black holes. This can explain why no IMBHs have yet been found $\mathrm{d}^{3}$, and it implies that present-day galaxies with $\sigma_{\star}<\mathcal{S}_{0} \approx 40 \mathrm{~km} \mathrm{~s}^{-1}$ lack a central black hole, or formed it only recently. A dearth of IMBHs at low redshifts has observable implications for tidal disruptions $^{8}$ and gravitational wave mergers?

The early stages of massive black hole growth are poorly understood ${ }^{10}$. High-luminosity active galactic nuclei at very high redshif ${ }^{[11} z$ further imply rapid growth soon after the Big Bang. Suggested formation mechanisms typically rely on the extreme conditions found in the early Universe (very low metallicity, very high gas or star density). It is therefore plausible that these black hole seeds were formed in dense environments, at least a Hubble time ago $\left(z>1.8 \text { for a look-back time of } t_{H}=10 \mathrm{Gyr}\right)^{122}$.

The relation $M_{\bullet}=M_{s}\left(\sigma_{\star} / \sigma_{s}\right)^{\beta}$ between black hole mass, $M_{\bullet}$, and stellar velocity dispersion, $\sigma_{\star}$, that is observed in the local Universe over more than about three decades in massive black hole mass, correlates $M_{\bullet}$ and $\sigma_{\star}$ on scales that are well outside the massive black hole's radius of dynamical influence ${ }^{\sqrt{3}}$, $r_{\mathrm{h}} \approx G M_{\bullet} / \sigma_{\star}^{2}$. Recent analyses of large heterogeneous galaxy samples find that a universal $M_{\bullet} / \sigma_{\star}$ relation holds for all galaxy types ${ }^{67}$, although the scope of this relation and its evolution with redshift remain controversial ${ }^{[13}$. Here we adopt the empirical fit ${ }^{7}$ $\log _{10}\left(M_{\bullet} / M_{\odot}\right)=8.32 \pm 0.04 \pm \delta_{\epsilon}+(5.35 \pm 0.23) \log _{10}\left(\sigma_{\star} / 200 \mathrm{~km} \mathrm{~s}^{-1}\right)$, where $\delta_{\epsilon}=0.49 \pm 0.03$ is the root mean square of the intrinsic scatter. We assume that this universal $M_{\bullet} / \sigma_{\star}$ holds at all redshifts $s^{14}$, and that the black hole seeds grow in a locally (within a few $r_{\mathrm{h}}$ ) dense stellar environment. By fixing $r_{\mathrm{h}}$, the $M_{\bullet} / \sigma_{\star}$ relation then imposes tight connections between the black hole and the dynamical properties of its stellar surrounding $\sqrt{15}^{15}$, and specifically the rate at which it consumes stars (see Methods section).

A central black hole grows by (1) the accretion of stars, compact remnants and dark matter particles that are deflected toward it on nearly radial orbits, and either fall whole through the event horizon or are tidally disrupted outside it, and then accreted; (2) viscosity-driven accretion of interstellar gas; and (3) mergers with other black holes. Of these growth channels, only the accretion of stars must follow from the existence of a central black hole in a stellar system. Moreover, the tidal disruption event

*Department of Particle Physics \& Astrophysics, Weizmann Institute of Science, Rehovot 76100, Israel.

${ }^{\dagger}$ Institute for Advanced Study, Einstein Drive, Princeton, NJ 08540, USA
(TDE) rate in steady-state can be estimated from first principles, for given boundary conditions at $r_{\mathrm{h}}$ (ref. $\left.{ }^{16}\right)$.

It has been noted that typical steady-state TDE rates, $\Gamma$, around $10^{-4} \mathrm{yr}^{-1}$ (Fig. 1), imply by simple dimensional analysis that massive black holes (MBHs) with low mass, $\lesssim 10^{7} M_{\odot}$, may acquire a substantial fraction of their mass from TDEs over the Hubble time $t_{\mathrm{H}}$, or equivalently, that linear growth by TDEs has a typical mass scale ${ }^{17719}, M_{\bullet}^{\mathrm{TDE}} \sim M_{\star} \Gamma t_{\mathrm{H}} \sim 10^{6} M_{\bullet}$ (however, the growth equation is generally nonlinear, and therefore $M_{\bullet}^{\mathrm{TDE}}$ can significantly mis-estimate $M_{\bullet}\left(t_{H}\right)$; see Methods section). Previous studies have usually focused on the rates and prospects of TDE detection, and not on black hole growth. Although it was recently argued that $M_{\bullet}^{\mathrm{TDE}}$ arises as a minimal black hole mass in a specific formation scenaric ${ }^{19}$, the commonly held assumption remains that IMBHs with $M_{\bullet} \ll M_{\bullet}^{\mathrm{TDE}}$ do exist, and that this must constrain formation scenarios, or set an upper bound on the efficiency of TDE accretion, rather than a lower bound on IMBH masse ${ }^{118}$.

Here, we argue that IMBHs are transient objects, which no longer exist in the present-day Universe. We derive a universal lower bound on the present-day mass scale of central black holes, $\mathcal{M}_{0}$, that follows directly from the universal $M_{\bullet} / \sigma_{\star}$ relation, and is independent of the unknown seed masses and their formation processes. We use the $M_{\bullet} / \sigma_{\star}$ relation to set the boundary conditions, and show that the nonlinear growth equation for black holes can be bounded from below by a simple inequality that includes only growth by TDEs. We translate the intrinsic scatter in the $M_{\bullet} / \sigma_{\star}$ relation to a probability distribution for the lower bounds $\mathcal{M}_{0}$ and $\mathcal{S}_{0}$, and show that $\mathcal{M}_{0}$ lies just below the lightest MBHs yet discovered ${ }^{3}$, $\mathcal{M}_{0} \lesssim \min \left(M_{\bullet}^{\text {obs }}\right) \sim 10^{6} M_{\odot}$.

Stars around a central black hole are constantly scattered in angular momentum to nearly radial orbits below a critical ("losscone") value, $j_{l c}=\sqrt{1-e^{2}}$ ( $e$ is the orbital eccentricity), which approach the black hole closer than the tidal disruption radius, $r_{t} \simeq\left(M_{\bullet} / M_{\star}\right)^{1 / 3} R_{\star}\left(M_{\star}\right.$ and $R_{\star}$ are the stellar mass and radius), where they are destroyed. Main sequence stars are disrupted outside an IMBH's event horizon, and a fraction $f_{a}$ of about $1 / 4$ to $1 / 2$ of their mass is ultimately accreted by the black hole ${ }^{20}$. The TDE rate depends on the number of stars near the black hole and on the competition between the twobody relaxation time $T_{R}$ (equation (12)) and the orbital time in supplying and draining loss-cone orbits. The integrated contribution in steady-state from all radii is a function of $M_{\bullet}$ and of the boundary conditions at $r_{\mathrm{h}}$, fixed by $\sigma_{\star}$. The TDE rate is wellapproximated by a power-law $\Gamma \simeq \Gamma_{\star}\left(M_{\bullet} / M_{\star}\right)^{b}$, whose index $b$ is a function of the $M_{\bullet} / \sigma_{\star}$ index $\beta$, and changes across a critical mass scale $M_{c} \sim 10^{6} M_{\odot}$ (Fig. 1) see Methods section). The index $b \ll 1$ for the empirical range $4 \lesssim \beta<6\left(\operatorname{refs} \frac{313}{113}\right)$.

Let us assume that a black hole seed forms with an initial mass $M_{i}$ large enough to dominate its radius of influence, in a central stellar system that is massive enough to allow it to grow: that is, $M_{\mathrm{sys}} \gg r_{\mathrm{h}}^{3} M_{\star} n_{\star}\left(r_{\mathrm{h}}\right)>M_{\bullet} \gg M_{\star}$ at all times $\left(n_{\star}\right.$ is the 


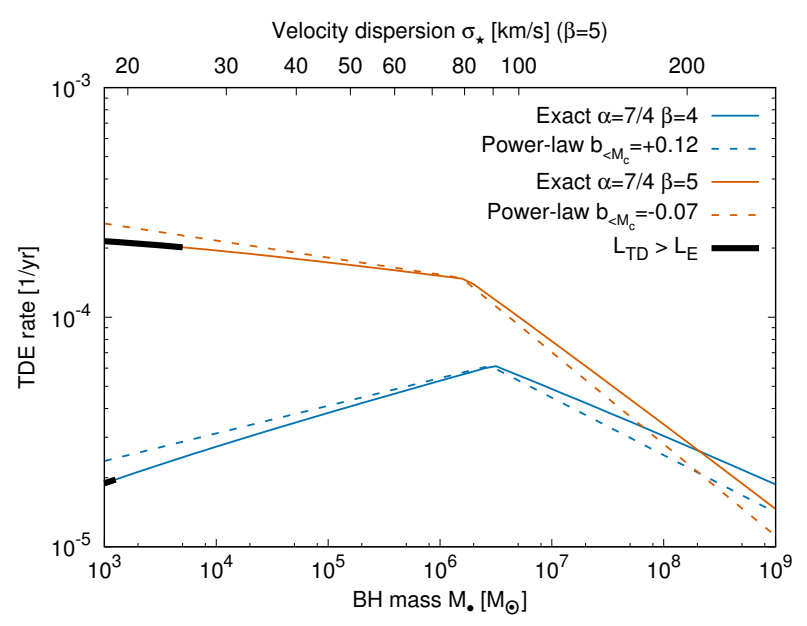

Figure 1 Plunge rates as function of the black hole mass $M_{\bullet}$. Rates (solid lines, see Methods section) are plotted for $M_{\star}=1 M_{\odot}$, $R_{\star}=1 R_{\odot}, f_{a}=3 / 8, \alpha=7 / 4$, and $\beta=4,5$, normalized to the empirical $M_{\bullet} / \sigma_{\star}$ mass parameter $M_{s}=2.1 \times 10^{8} M_{\odot}{ }^{7}$. The corresponding velocity dispersion $\sigma_{\star}$ is displayed for a $\beta=5 M_{\bullet} / \sigma_{\star}$ relation. The rates are well-approximated by power-laws (dashed lines, equation (4)) Radiation back-reaction above the Eddington limit $L_{E}$ by the mean accretion luminosity $L_{T D}=\eta f_{a} M_{\star} c^{2} \Gamma(\eta=0.1$ assumed for the radiative efficiency) is only relevant for the lowest black hole masses, where it may slow down the initial black hole growth.

stellar density). Consider first the case where the black hole grows only by accreting stars. The black hole growth equation is

$$
\dot{M}_{\bullet}=f_{a} M_{\star} \Gamma_{\star}\left(M_{\bullet} / M_{\star}\right)^{b} \equiv \dot{M}_{\bullet}^{\star}, \quad M_{\bullet}(0)=M_{i} .
$$

The solution for $b<1$ in the $t \gg t_{\infty}=\left(M_{i} / M_{\star}\right)^{1-b} /\left(|1-b| f_{a} \Gamma_{\star}\right) \quad$ limit (equation (9)), $M_{\bullet}(t) \simeq\left[(1-b) f_{a} \Gamma_{\star} t\right]^{1 /(1-b)} M_{\star} \equiv M_{\bullet}^{\star}(t)$, is independent of $M_{i}$. Because $t_{\infty} \ll t_{H}$ for $M_{i} \lesssim 10^{5} M_{\odot}$, all seeds reach the same mass scale after $\mathcal{O}\left(t_{H}\right)$ (Fig.2. 2 .

Consider next a realistic black hole that grows also by gas accretion and/or mergers, $\dot{M}_{\bullet}=\dot{M}_{\bullet}^{\star}+\dot{M}_{\bullet}^{+}$, where $\dot{M}_{\bullet}^{+} \geq 0$ is the accretion rate by the non-stellar channels. The full growth equation is

$$
\dot{M}_{\bullet}=\dot{M}_{\bullet}^{\star}+\dot{M}_{\bullet}^{+} \geq f_{a} M_{\star} \Gamma_{\star}\left(M_{\bullet} / M_{\star}\right)^{b}, \quad M_{\bullet}(0)=M_{i} .
$$

The solution $M_{\bullet}^{\star}(t)$ of the stars-only growth (equation (1)) then provides a lower limit on the actual mass $M_{\bullet}(t)$ of the growing black hole. Note that $M_{\bullet}^{\star}$ is not necessarily a lower limit on the actual stellar mass contribution $M^{\star}$ to $M_{\bullet}$ : $M_{\bullet}^{\star} \leq M^{\star} \leq M_{\bullet}$ for $b \geq 0$, but $M^{\star}<M_{\bullet}^{\star} \leq M_{\bullet}$ for $b<0$. The universal minimal mass scale of a central black hole at $t_{H}$, and the corresponding minimal velocity dispersion scale are then

$$
\mathcal{M}_{0}=M_{\bullet}^{\star}\left(t_{H}\right)=\left[(1-b) f_{a} \Gamma_{\star} t_{H}\right]^{1 /(1-b)} M_{\star}, \mathcal{S}_{0}=\left(\mathcal{M}_{0} / M_{s}\right)^{1 / \beta} \sigma_{s},
$$

with the index $b$ for the $M_{\bullet}<M_{c}$ branch of equation (6). This implies that galaxies with $\sigma_{\star}<\mathcal{S}_{0}$ do not have a central black hole, or have formed it only recently, for otherwise the coevolution of the black hole and nucleus over $t_{H}$ would have driven $\sigma_{\star}$ to a much larger present-day value.

Assuming the universal $M_{\bullet} / \sigma_{\star}$ relation and Solar type stars, the range in $\mathcal{M}_{0}$ values is due to the variety and uncertainty in

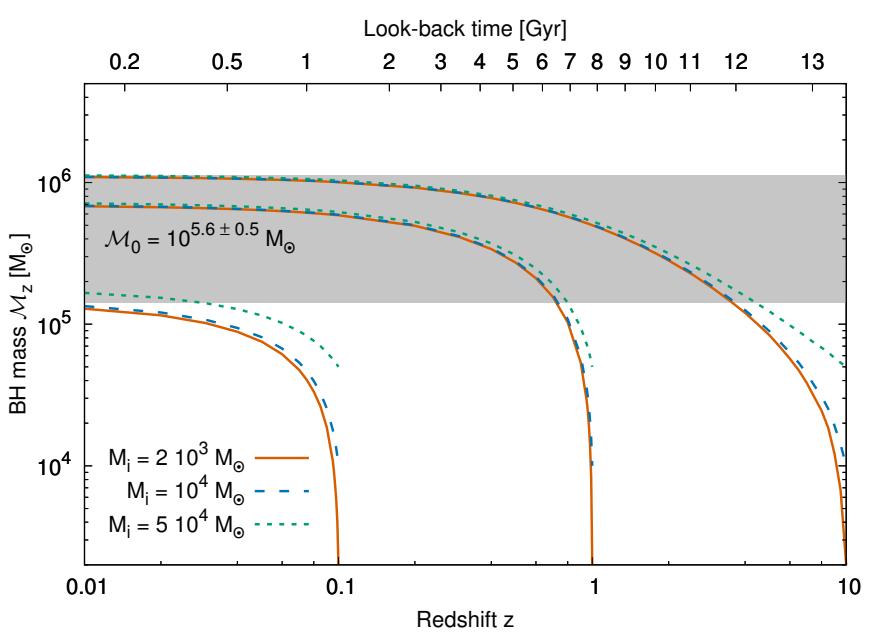

Figure 2 Cosmological growth of the minimal black hole mass $\mathcal{M}_{z}$ as function of redshift. The evolution of the minimal black hole mass (equation (10) ) is plotted for several values of the black hole seed mass $M_{i}$ at different formation redshifts $z_{i}$ (look-back times ${ }^{12} t_{i}$ ), assuming the empirical $M_{\bullet} / \sigma_{\star}$ relatior ${ }^{7]}$ without scatter, an $\alpha=7 / 4$ cusp of Solar type stars and an accreted mass fraction $f_{a}=3 / 8$. The convergence to evolution that is independent of initial mass is rapid in redshift. The earlier the formation, the higher is $\mathcal{M}_{0}$. The range $z_{i}=0.1-10$ translates to a lower mass limit on present-day MBHs $\mathcal{M}_{0} \sim 10^{(5.6 \pm 0.5) M_{\odot}}$ (gray band).

the properties of galactic nuclei and of tidal disruption, and to the intrinsic scatter in the relation. $\mathcal{M}_{0}$ increases with the index $\alpha$ of the stellar cusp $\left(n_{\star} \propto r^{-\alpha}\right)$ inside $r_{\mathrm{h}}$, and with the accreted mass fraction $f_{a}$, from $10^{5} M_{\odot}$ for the shallowest possible cusp of unbound stars $\left(\alpha=1 / 2, f_{a}=1 / 4\right)$ to $10^{6} M_{\odot}$ for a steep isothermal cusp $\left(\alpha=2, f_{a}=1 / 2\right) . \mathcal{M}_{0} \simeq 10^{6} M_{\odot}$ for the parameters adopted here: a dynamically relaxed cusp ${ }^{211}$ where $\alpha=7 / 4$, and an accreted mass fraction $f_{a}=3 / 8$.

The scatter around the $M_{\bullet} / \sigma_{\star}$ relation likely reflects intrinsic physical differences between galaxies beyond the measurement errors on the $M_{\bullet} / \sigma_{\star}$ parameters ${ }^{7}$. This induces roughly Gaussian probability distributions for $\mathcal{M}_{0}$ and $\mathcal{S}_{0}: \mathcal{M}_{0}=(1.1 \pm 0.8) \times 10^{6} M_{\odot}$ and $\mathcal{S}_{0}=79 \pm 35 \mathrm{~km} \mathrm{~s}^{-1}$ $(1 \sigma)$. The lowest- $\sigma_{\star}$ galaxies known to harbor active galactic nuclei ${ }^{22]}$ (and hence black holes, with estimated masses $\left.10^{5} \lesssim M_{\bullet} \lesssim 10^{6} M_{\odot}\right)$, have $\sigma_{\star} \sim 30-40 \mathrm{~km} \mathrm{~s}^{-1}$. This corresponds to the $5 \%$ lower limits $\mathcal{S}_{0} \lesssim 40 \mathrm{~km} \mathrm{~s}^{-1}$ and $\mathcal{M}_{0} \lesssim 3 \times 10^{5} M_{\odot}$, which we adopt here as representative lower limits. Lighter black holes are much rarer yet: e.g. $M_{\bullet} \leq 10^{4} M_{\odot}$ is below the $0.0001 \%$ limit.

The agreement $\mathcal{M}_{0} \lesssim \min \left(M_{\bullet}^{\text {obs }}\right) \sim 10^{6} M_{\odot}$ follows directly from basic local physics (tidal disruption and loss-cone dynamics) and empirical global properties of the Universe (its age and a universal $M_{\bullet} / \sigma_{\star}$ relation). Our derivation of $\mathcal{M}_{0}$ rests on four assumptions. (1) There is effective accretion of tidally disrupted stars $\left(f_{a} \text { is a few } \times 0.1\right)^{20}$. (2) Most black hole seeds were formed early, at a look-back time $t_{i} \sim \mathcal{O}\left(t_{H}\right)$. (3) Black hole growth is not typically mass or density limited; that is, the growing black hole is embedded in a stellar system with $M_{\text {sys }} \gg r_{\mathrm{h}}^{3} M_{\star} n_{\star}>M_{\bullet}$ for a substantial fraction of $t_{H}$. (4) The boundary conditions at $r_{\mathrm{h}}$ are set by the universal $M_{\bullet} / \sigma_{\star}$ relation at all times.

An early start for black hole seeds, and the requirement that a system that can form and retain a seed black hole 
should be dense and massive enough, are both physically plausible and possibly even essential10. Such a system can be approximated as embedded in an isothermal density distribution, and is dynamically relaxed (see Methods section). Furthermore, the accretion rate of stars in a system with $N_{\star}$ stars inside $r_{\mathrm{h}}, \mathrm{d} M_{\bullet} / \mathrm{dt} \simeq f_{a} M_{\star} N_{\star} /\left(\log \left(1 / j_{l c}\right) T_{R}\right)$ (equation (16)), is slow enough to allow it to remain near equilibrium as it grows, as the timescale for growth by order of the stellar mass, $\left(\mathrm{d} M_{\bullet} / \mathrm{dt}\right) /\left(M_{\star} N_{\star}\right)$, is longer by a factor $\log \left(1 / j_{l c}\right) / f_{a} \gg 1$ than the timescale to return to steady-state, $T_{S S} \simeq T_{R} / 4$ (ref. ${ }^{21}$ ). The least secure assumption is that a universal $M_{\bullet} / \sigma_{\star}$ relation holds near its present-day value as the black hole grows. However, this is broadly consistent with observations of active galactic nuclei $i^{\sqrt{14 / 23}}$ up to $z \sim 1$, and with simulations of large scale structure evolution ${ }^{24 \mid 25}$ up to $z \sim 4$.

Mergers between two cental black holes increase the black hole mass, but also affect the dynamics around it. Mergers initially enhance the TDE rate ${ }^{\sqrt{26}}$, but later they may strongly suppress it by ejecting the cusp. However, steady-state is quickly re-established around IMBHs, $T_{S S}\left(M_{\bullet} \lesssim 10^{5} M_{\odot}\right)<\mathcal{O}\left(10^{8} \mathrm{yr}\right)$ (ref. ${ }^{21}$ ). Additional growth channels thus only increase present-day black hole masses, and reinforce the conclusion that central black holes with $M_{\bullet}<\mathcal{M}_{0}$ are rare.

Figure 2 shows the evolution of the lower mass limit $\mathcal{M}_{z}$, which increases rapidly with decreasing redshift to its presentday value $\mathcal{M}_{0}$. Present-day IMBHs may exist in recently formed systems, in mass-limited ones (for example globular clusters with $M<10^{6} M_{\odot}$ ), in sub-galactic systems where the $M_{\bullet} / \sigma_{\star}$ relation need not apply (such as globular clusters or super star clusters), or in very low density galaxies (such as cored dwarfs $\left.{ }^{27}\right)$. However, it is unlikely that such systems can form a black hole seed to start with ${ }^{1 / 10}$, and therefore the black hole occupation fraction there is probably low. Candidate IMBHs have been reported in globular clusters and dwarf galaxies, including recently $\sqrt{2829}$, but the evidence remains inconclusive.

Early TDE-driven growth and the suppression of the cosmic black hole mass function below $\mathcal{M}_{0}$ have implications for black hole seed evolution, for the cosmic rates and properties of $\mathrm{TDEs}^{8}$, and for gravitational waves (GWs) from IMBH-IMBH mergers and intermediate-mass-ratio inspirals into IMBHs. We conclude by listing these briefly.

A high rate of TDEs can allow black hole seeds to continue growing despite the ejection of the ambient gas by supernovae feedback $^{30}$. The lack of IMBHs at low redshifts means that electromagnetic searches will have to reach very deep to detect TDEs from IMBHs (jetted TDEs may provide an opportunity ${ }^{31}$ ). The prospects of detecting exotic processes related to IMBHs, such as tidal detonations of white dwarfs in the steep tidal field of a low-mass black hole ${ }^{32}$, will be low. The mean observed TDE rate per galaxy, $\Gamma \sim 10^{-5} \mathrm{yr}^{-1} \mathrm{gal}^{-1}$, is much lower than predicted rates ${ }^{33}$. A dearth of black holes below $\mathcal{M}_{0}$ may partially resolve the rate discrepancy.

IMBHs produce GWs by intermediate-mass-ratio inspirals and by IMBH mergers. Detection of intermediate-mass-ratio inspirals by planned space-borne $\mathrm{GW}$ observatories ${ }^{9{ }^{93}}$ is limited to redshifts below a few $\times 0.1$, and is therefore unlikely. However, IMBH mergers can be detected to very high redshifts. A GW search for IMBH mergers and intermediate-mass-ratio inspirals can reveal the formation history of black holes. We predict that black hole seeds are driven early on to higher mass by the ac- cretion of stars, and therefore IMBHs are rare in the present-day Universe, but will be found near their high formation redshifts.

\section{Methods}

This supplement summarizes results from loss-cone theory used to derive the equation for black hole growth by stellar disruptions, and discusses the properties of its solutions. We first present, without derivation, a recipe for the approximate power-law growth rate equation (equation (10), which has the advantage of leading to simple analytic results. We then comment on the general properties of its solutions, to clarify under what circumstances, and to what extent can simple dimensional analysis be used to estimate the minimal mass limit $\mathcal{M}_{0}$. We then describe how the intrinsic scatter in the $M_{\bullet} / \sigma_{\star}$ relation is propagated through the growth equation to obtain the probability distributions for the lower limits $\mathcal{M}_{0}$ and $\mathcal{S}_{0}$. Finally, we present for completeness and reproducibility an outline of the derivation of the full growth rate equation (used to verify our approximations, see Figure 11) and of its power-law approximation.

Approximate power-law black hole growth rate equation. We focus here on a steady-state stellar system around a black hole ${ }^{35}$, which has a density cusp $n_{\star} \propto r^{-\alpha}$ with $\alpha=7 / 4$ inside the radius of influence $r_{\mathrm{h}}=G M_{\bullet} / \sigma_{\star}^{2}$. We further assume that the cusp is embedded in an external isothermal stellar distribution, $\rho(r)=\sigma_{\star}^{2} /\left(2 \pi G r^{2}\right)$, so that the stellar mass enclosed inside $r_{\mathrm{h}}$ is twice the black hole mass ${ }^{18}$. Under the assumption of a universal $M_{\bullet} / \sigma_{\star}$ relation $M_{\bullet}=M_{s}\left(\sigma_{\star} / \sigma_{s}\right)^{\beta}$, the dynamics leading to tidal disruption are characterized by a critical mass scale $M_{c} \sim 10^{6} M_{\odot}$. Tidal disruptions are dominated by stars originating from $\sim r_{\mathrm{h}}\left(M_{\bullet}\right)$ for $M_{\bullet} \geq M_{c}$, and by stars originating from an inner critical radius $r_{c}\left(M_{\bullet}\right)<r_{\mathrm{h}}\left(M_{\bullet}\right)$ for $M_{\bullet}<M_{c}$ (see below for more details) ${ }^{\sqrt{36}}$. The TDE rate is well-approximated by a broken power-law (Figure 1 )

$$
\Gamma \simeq \Gamma_{\star}\left(M_{\bullet} / M_{\star}\right)^{b},
$$

whose index $b$ changes across $M_{c}$, which is given by (see equations 20, 21 for the general case),

$$
M_{c} \simeq M_{\star}\left(\frac{16}{5 s^{2}}\right)^{3 \beta /(6+\beta)},
$$

in terms of the dimensionless velocity dispersion scale $s=\left(M_{s} / M_{\star}\right)^{-1 / \beta} \sigma_{s} / v_{\star}$, where $v_{\star}^{2}=G M_{\star} / R_{\star} \quad$ and $M_{\star}$ and $R_{\star}$ are the mass and radius of a typical star in the system, and where we approximated the logarithmic term (equation (14) appearing in the general expressions by a typical value $\Lambda_{l c}=2$. The index $b$ is (see equation (22) for the general case),

$$
b=\left\{\begin{array}{ll}
(105-23 \beta) / 27 \beta & M_{\bullet} \leq M_{c} \\
(3-\beta) / \beta & M_{\bullet}>M_{c}
\end{array} .\right.
$$

Note that $b \ll 1$ for the empirically determined range of the $M_{\bullet} / \sigma_{\star}$ relation index $\sqrt{3 \sqrt{13}}, 4 \lesssim \beta<6$. Defining $t_{\star}=\sqrt{R_{\star}^{3} / G M_{\star}}$, the rate factor is

$$
\Gamma_{\star} \simeq \frac{5 / 4}{t_{\star}} s^{40 / 9} \begin{cases}1 & M_{\bullet} \leq M_{c} \\ \left(M_{c} / M_{s}\right)^{4(6+\beta) / 27 \beta} & M_{\bullet}>M_{c}\end{cases}
$$

To summarize, the approximate power-law TDE rate for a black hole with mass $M_{\bullet}$ is calculated as follows. (1) Calculate the critical mass $M_{c}$ (equation (5)). (2) Calculate the power-law index $b$ (equation (6) according to the low or high mass branch, depending on $M_{\bullet}$, and similarly calculate the rate factor $\Gamma_{\star}$ (equation (7)). (3) Use equation (4) to obtain the TDE rate from $\Gamma_{\star}$ and $b$. 
Properties of the black hole growth solutions. The general solution of the growth equation (equation (4)) with the initial condition $M_{\bullet}(t=0)=M_{i}$ is

$$
M_{\bullet}(t)=\left\{\begin{array}{ll}
M_{\star}\left[\left(M_{i} / M_{\star}\right)^{1-b}+(1-b) t / t_{a}\right]^{1 /(1-b)} & b \neq 1 \\
M_{i} e^{t / t_{a}} & b=1
\end{array},\right.
$$

where $t_{a}=\left(f_{a} \Gamma_{\star}\right)^{-1}$ is the accretion timescale. The growth solution has three branches. The solution for $b=1$ diverges exponentially to infinity in infinite time. When $b>1, M_{\bullet}$ diverges on a finite timescale

$$
t_{\infty}=t_{a}\left(M_{i} / M_{\star}\right)^{1-b} /|1-b|,
$$

and is supra-exponential. The $b<1$ branch is sub-exponential and diverges slowly as a power-law.

Exponential growth describes, for example, radiation pressureregulated accretion of gas at the Eddington limit. Supra-exponential growth describes Hoyle-Lyttleton wind accretior ${ }^{37}$, spherical Bondi accretion ${ }^{38}$, or their generalization of accretion on an accelerating black hole ${ }^{39}$. Sub-exponential growth, which is the relevant case for tidal accretion with the universal $M_{\bullet} / \sigma_{\star}$ relation, at $t \gg t_{\infty}$ asymptotically approaches a power law that is independent of seed mass $M_{i}$

$$
M_{\bullet}(t) / M_{\star} \simeq\left[(1-b) t / t_{a}\right]^{1 /(1-b)} .
$$

There are two mass scales in the growth equation: the initial mass $M_{i}$, and the natural mass scale ${ }^{40 \mid 41} M^{\text {TDE }}$, obtained by solving $M^{\mathrm{TDE}} / M_{\star}=f_{a} \Gamma t_{H}=f_{a} \Gamma_{\star} t_{H}\left(M^{\mathrm{TDE}} / M_{\star}\right)^{b}$ with $t_{H}=10^{10}$ yr. This mass scale was used in past studies $s^{\sqrt{17,18,33}}$ to estimate $M_{\bullet}\left(t_{H}\right)$. It is instructive to analyze the role of $M_{\bullet}^{\mathrm{TDE}}$ in the growth solutions, and identify when it can provide a relevant estimate for the black hole mass.

The exponential and supra-exponential solutions $(b \geq 1)$ are functions of $M_{i}$ on all timescales, and $M_{\bullet}^{\mathrm{TDE}}$ plays there a role related to the exponential or divergence timescales. Because these solutions diverge, the black hole mass at any finite time is generally unrelated to either $M_{i}$ or $M_{\bullet}^{\mathrm{TDE}}$. The asymptotic sub-exponential solution $(b<1)$ can be written as $M_{\bullet}\left(t_{H}\right)=M_{\bullet}^{\mathrm{TDE}}(1-b)^{1 /(1-b)}$. In this case, $M_{\bullet}^{\mathrm{TDE}}$ provides a reasonable approximation for $M_{\bullet}$ as long as $|b| \ll 1$. This is the indeed case for the empirical universal $M_{\bullet} / \sigma_{\star}$ relation, where $b(\alpha=7 / 4, \beta=5.40) \simeq-0.125$. However, other combinations of cusp and $M_{\bullet} / \sigma_{\star}$ indices can lead to arbitrarily large disparities: for example $b(\alpha=3 / 2, \beta=3) \simeq 0.6$, results in $M_{\bullet} \simeq 0.1 M_{\bullet}^{\mathrm{TDE}}$.

It should be emphasized that the solution branch that describes the black hole growth is not determined solely by the assumed growth channel - tidal disruptions in this case - but also by the choice of boundary conditions, which here are determined by an empirical relation. Other possible values of cusp and $M_{\bullet} / \sigma_{\star}$ indices would imply very different relations between $M_{\bullet}$ and $M_{\bullet}^{\mathrm{TDE}}$. For example, the transition to the exponential and supra-exponential solutions $(b \geq 1$ ) occurs for $\beta \leq 2.1$ (for $\alpha=7 / 4$ ) or for $\beta \leq 3$ (for $\alpha=1 / 2)$. Therefore, it is not generally true that $M_{\bullet}^{\mathrm{TDE}}$ estimates the black hole mass. Its relevance depends on the specific solution and on the adopted boundary conditions, and cannot be assumed a priori.

Intrinsic $M_{\bullet} / \sigma_{\star}$ scatter and distribution of lower limits. The observed intrinsic scatter in the $M_{\bullet} / \sigma_{\star}$ relation at $z \simeq 0$, with r.m.s $\delta_{\epsilon}$, can be interpreted as reflecting a variance in the initial conditions of individual galaxies at their formation, a Hubble time $t_{H}$ ago, or a variance that developed gradually over their individual evolutionary histories and reached the observed rms value at $t_{H}$.

We assume that the estimation errors in the parameters $\alpha$ and $\beta$ of the $M_{\bullet} / \sigma_{\star}$ relation, $\log \left(M_{\bullet} / M_{\star}\right)=\left(\bar{\alpha} \pm \delta_{\alpha}\right)+\left(\bar{\beta} \pm \delta_{\beta}\right) \log \left(\sigma / \sigma_{s}\right) \pm \delta_{\epsilon}$, can be approximated by a correlated bi-Gaussian distribution, $(\alpha, \beta) \sim G_{2}\left(\bar{\alpha}, \delta_{a}, \bar{\beta}, \delta_{\beta}, \rho_{\alpha \beta}\right)$, where for an arbitrarily chosen low reference velocity dispersion $\sigma_{s} \ll \sigma$, the correlation coefficient $\rho_{\alpha \beta} \rightarrow-1$ (ref. ${ }^{42}$ ), and that the intrinsic scatter $\epsilon$ is drawn from a Gaussian distribution, $\epsilon \sim G\left(0, \delta_{\epsilon}\right)$.

We approximate the evolution of the scatter by assuming $n_{t}$ discrete time steps of duration $\Delta t=t_{H} / n_{t}$, where the accumulated change in $\alpha$ due to scatter, $\Delta \epsilon$, is modified in a random walk fashion by $\Delta \epsilon \rightarrow \Delta \epsilon+\epsilon / \sqrt{n_{t}}$. We then evolve the black hole mass over time $\Delta t$ by the growth equation (equation (8)), and repeat until $t=t_{H}$. The joint and marginal probability distributions for $\mathcal{M}_{0}$ and $\mathcal{S}_{0}$ at $t_{H}$ are obtained by Monte Carlo simulations over randomly drawn values of $\alpha$ and $\beta$.

The limit $n_{t}=1$ corresponds to scatter that is determined by the galaxy's initial conditions, whereas $n_{t} \gg 1$ corresponds to scatter that is determined by the galaxy's evolution. We find that the probability distributions for $\mathcal{M}_{0}$ and $\mathcal{S}_{0}$ do not depend strongly on the choice of $n_{t}$, and that they converge rapidly for $n_{t}>3$ to an asymptotic form. The values quoted in this study, $5 \%$ lower limits of $\mathcal{M}_{0}=2.8 \times 10^{5} M_{\odot}$ and $\mathcal{S}_{0}=38 \mathrm{~km} \mathrm{~s}^{-1}$, correspond to the asymptotic evolutionary scatter case $\left(n_{t}=5\right)$, whereas the initial scatter case $\left(n_{t}=1\right)$ differs only slightly, with $5 \%$ lower limits of $\mathcal{M}_{0}=1.9 \times 10^{5} M_{\odot}$ and $\mathcal{S}_{0}=36 \mathrm{~km} \mathrm{~s}^{-1}$.

Full black hole growth rate equation. The tidal disruption (plunge) rate can be approximated by the flux of stars into the black hole from from the boundary between the inner region, where stars slowly diffuse into the loss-cone (the empty loss-cone) and the outer region, where stellar scattering is strong enough that the loss-cone is effectively full (the full loss-cone $)^{\sqrt{36}}$. The boundary is at a critical radius, $a_{c}$, that satisfies

$$
q=\frac{\left[J_{c}\left(a_{c}\right) / J_{l c}\right]^{2} P\left(a_{c}\right)}{\log \left(J_{c}\left(a_{c}\right) / J_{l c}\right) T_{R}\left(a_{c}\right)}=1
$$

where $P=2 \pi \sqrt{a^{3} / G M_{\bullet}}$ is the orbital period, $J_{c}=\sqrt{G M_{\bullet} a}$ is the circular angular momentum at $a, J_{l c}$ is angular momentum of the loss-cone $\left(J_{l c} \simeq \sqrt{2 G M_{\bullet} Q^{1 / 3} R_{\star}}\right.$ for tidal disruption, so $J_{c} / J_{l c} \simeq \sqrt{\left(a / R_{\star}\right) / 2 Q^{1 / 3}}$, where $\left.Q=M_{\bullet} / M_{\star}\right) . \quad T_{R}$ is the 2body (non-resonant) relaxation time ${ }^{[21}$,

$$
T_{R}(a)=\frac{5}{8} \frac{Q^{2} P(a)}{N_{\star}(a) \log (Q)},
$$

where $N_{\star}(a)=\mu_{h} Q\left(a / r_{\mathrm{h}}\right)^{3-\alpha}$ is the number of stars enclosed in $r$, and $r_{\mathrm{h}}=\eta_{h} G M_{\bullet} / \sigma_{\star}^{2}$ is the radius of influence. The numeric prefactors are conventionally assumed to be $\mu_{h}=2$ and $\eta_{h}=1$. We further assume that $\alpha<9 / 4$.

The exact solution for the critical radius can be written by the implicit equation

$$
\begin{aligned}
a_{c} / r_{\mathrm{h}} & =\left(A_{c} \sigma_{\star}^{2} / v_{\star}^{2}\right)^{1 /(4-\alpha)} Q^{1 /(12-3 \alpha)} \\
& =\left(A_{c} s^{2}\right)^{1 /(4-\alpha)} Q^{(\beta+6) / \beta(12-3 \alpha)},
\end{aligned}
$$

where $A_{c}=5 /\left(4 \mu_{h} \Lambda_{l c} \eta_{h}\right)$ and

$$
\Lambda_{l c}(Q, a)=\log Q / \log \left(J_{c}(a) / J_{l c}\right),
$$

where the last equality in equation (13) assumes the $M_{\bullet} / \sigma_{\star}$ relation in terms of the dimensionless velocity dispersion scale $s=\left(M_{s} / M_{\star}\right)^{-1 / \beta} \sigma_{s} / v_{\star}$, where $v_{\star}=\sqrt{G M_{\star} / R_{\star}}$.

When $a_{c}>r_{\mathrm{h}}$, the rate is estimated ${ }^{433}$ at $r_{\mathrm{h}}$. The transition occurs above a critical black hole mass such that $a_{c}\left(M_{c}\right)=r_{\mathrm{h}}\left(M_{c}\right)$,

$$
M_{c}=M_{\star}\left(A_{c} \sigma_{\star}^{2} / v_{\star}^{2}\right)^{-3}=M_{\star}\left(A_{c} s^{2}\right)^{-3 \beta /(6+\beta)},
$$

which is independent of $\alpha$ and almost independent of $\beta$. The plunge rate can then be conservatively approximated by the empty loss-cone rate at $a_{e}=\min \left(a_{c}, r_{\mathrm{h}}\right)$,

$$
\Gamma \simeq \frac{N_{\star}\left(a_{e}\right)}{\log \left(J_{c}\left(a_{e}\right) / J_{l c}\right) T_{R}\left(a_{e}\right)}=\frac{8}{5} \frac{\Lambda_{l c} \mu_{h}^{2}}{P\left(r_{\mathrm{h}}\right)}\left(\frac{a_{e}}{r_{\mathrm{h}}}\right)^{(9-4 \alpha) / 2} .
$$


The actual rate, including the contribution from the full loss-cone regime, can be up to twice as high as this as this ${ }^{43}$.

Using $r_{\mathrm{h}}=\eta_{h} G M_{\bullet} / \sigma_{\star}^{2}$, the plunge rate can be represented as

$$
\Gamma^{\Lambda}=\frac{1}{\pi t_{\star}}\left\{\begin{array}{ll}
\gamma_{c}^{\Lambda} Q^{(2 \alpha-15) / 6(4-\alpha)}\left(\frac{\sigma_{\star}}{v_{\star}}\right)^{7(3-\alpha) /(4-\alpha)} & M_{\bullet} \leq M_{c} \\
\gamma_{h}^{\Lambda} Q^{-1}\left(\frac{\sigma_{\star}}{v_{\star}}\right)^{3} & M_{\bullet}>M_{c}
\end{array},\right.
$$

where $t_{\star}=\sqrt{R_{\star}^{3} / G M_{\star}}$,

$$
\gamma_{c}^{\Lambda}=\left(\frac{4 \Lambda_{l c}}{5}\right)^{(2 \alpha-1) /(8-2 \alpha)}\left(\frac{\mu_{h}}{\eta_{h}^{(3-\alpha)}}\right)^{7 /(8-2 \alpha)},
$$

and

$$
\gamma_{h}^{\Lambda}=\frac{4 \mu_{h}^{2} \Lambda_{l c}}{5 \eta_{h}^{3 / 2}}
$$

When $\sigma_{\star}$ is given by the $M_{\bullet} / \sigma_{\star}$ relation, the rate can be expressed as

$$
\Gamma=\Gamma_{\star}^{\Lambda} Q^{b}
$$

where

$$
\Gamma_{\star}^{\Lambda}=\frac{\gamma_{c}^{\Lambda}}{\pi t_{\star}} s^{7(3-\alpha) /(4-\alpha)}\left\{\begin{array}{ll}
1 & M_{\bullet} \leq M_{c} \\
\left(\frac{M_{c}}{M_{\star}}\right)^{(6+\beta)(9-4 \alpha) / 6(4-\alpha) \beta} & M_{\bullet}>M_{c}
\end{array} .\right.
$$

The notation $\Gamma_{\star}^{\Lambda}$ denotes the weak functional dependence on $Q$ via the logarithmic term $\Lambda_{l c} \simeq 2$. The index is

$$
b= \begin{cases}7(3-\alpha) / \beta(4-\alpha)-(15 / 2-\alpha) / 3(4-\alpha) & a_{c} \leq r_{\mathrm{h}} \\ (3-\beta) / \beta & a_{c}>r_{\mathrm{h}}\end{cases}
$$

A simpler power-law approximation can be obtained by choosing this typical value for the logarithmic term $\Lambda_{l c} \simeq 2$. Then, $\Gamma \simeq \Gamma_{\star} Q^{b}$ (see above), where the normalization $\Gamma_{\star}=\Gamma_{\star}^{\Lambda}\left(\Lambda_{l c}=2\right)$ is not a function of $Q$.

Figure 11 shows the TDE rates for $\mu_{h}=2, \eta_{h}=1$ in the two dynamical regimes $a_{c}<r_{\mathrm{h}}$ and $a_{c}>r_{\mathrm{h}} \quad\left(Q<Q_{c}\right.$ and $\left.Q>Q_{c}\right)$. At the lower mass end, the mean mass accretion rate may rise above the Eddington rate. For example, for $\beta=5$, $L_{T D} / L_{E} \lesssim 6$ at $M_{\bullet}=10^{3} M_{\odot}$, but falls below $L_{T D} / L_{E}=1$ for $M_{\bullet}>5 \times 10^{3} M_{\odot}$. Depending on the exact value of the logarithmic slope of the $M_{\bullet} / \sigma_{\star}$ relation, $\beta$, the TDE rate on the low-mass branch, can either rise or fall with $M_{\bullet}$.

Data availability statement The numeric results that support the plots within this paper and other findings of this study are available from the corresponding author upon reasonable request.

Acknowledgements We are grateful for helpful discussions with $Y$ Alexander, J. Gair, A. Gal-Yam, J. Green, J. Guillochon, M. MacLeod, N. Neumayer, T. Piran, E. Rossi, A. Sesana, J. Silk, N. Stone and B. Trakhtenbrot. TA acknowledges support by the I-CORE Program of the Planning and Budgeting Committee and The Israel Science Foundation (grant No 1829/12). BB acknowledges support by NASA (grant NNX14AM24G) and by the NSF (grant AST-1406166).

Author Contributions TA and BB developed the ideas presented in this paper together and collaborated in its writing.

Correspondence Correspondence and requests for materials should be addressed to Tal Alexander (tal.alexander@weizmann.ac.il) or Ben Bar-Or (benbaror@ias.edu).

Competing Interests The authors declare that they have no competing financial interests.
1. Miller, M. C. \& Colbert, E. J. M. Intermediate-Mass Black Holes. International Journal of Modern Physics D 13, 1-64 (2004).

2. Abbott, B. P. et al. Observation of gravitational waves from a binary black hole merger. Phys. Rev. Lett. 116, 061102 (2016).

3. Graham, A. W. Galaxy Bulges and Their Massive Black Holes: A Review. In Laurikainen, E., Peletier, R. \& Gadotti, D. (eds.) Galaxy Bulges, vol. 418 of Astrophysics and Space Science Library, 263313 (Springer International Publishing Switzerland, 2016).

4. Ferrarese, L. \& Merritt, D. A Fundamental Relation between Supermassive Black Holes and Their Host Galaxies. Astrophys. J. 539, L9-L12 (2000).

5. Gebhardt, K. et al. A Relationship between Nuclear Black Hole Mass and Galaxy Velocity Dispersion. Astrophys. J. 539, L13-L16 (2000).

6. McConnell, N. J. \& Ma, C.-P. Revisiting the Scaling Relations of Black Hole Masses and Host Galaxy Properties. Astrophys. J. 764, 184 (2013).

7. van den Bosch, R. C. E. Unification of the fundamental plane and Super Massive Black Hole Masses. Astrophys. J. 831, 134 (2016).

8. Rees, M. J. Tidal disruption of stars by black holes of 10 to the 6 th-10 to the 8th solar masses in nearby galaxies. Nature 333, 523-528 (1988)

9. Amaro-Seoane, P. et al. eLISA: Astrophysics and cosmology in the millihertz regime. GW Notes, Vol. 6, p. 4-110 6, 4-110 (2013).

10. Volonteri, M. The Formation and Evolution of Massive Black Holes. Science 337, 544 (2012).

11. Mortlock, D. J. et al. A luminous quasar at a redshift of $z=7.085$. Nature 474, 616-619 (2011).

12. Bennett, C. L., Larson, D., Weiland, J. L. \& Hinshaw, G. The $1 \%$ Concordance Hubble Constant. Astrophys. J. 794, 135 (2014).

13. Kormendy, J. \& Ho, L. C. Coevolution (Or Not) of Supermassive Black Holes and Host Galaxies. Ann. Rev. Astron. Astrophys. 51, 511-653 (2013).

14. Shen, Y. et al. The Sloan Digital Sky Survey Reverberation Mapping Project: No Evidence for Evolution in the $M_{\bullet}-\sigma_{*}$ Relation to $z \sim 1$. Astrophys. J. 805, 96 (2015).

15. Alexander, T. Key Questions about Galactic Center Dynamics. In Morris, M. R., Wang, Q. D. \& Yuan, F. (eds.) The Galactic Center: a Window to the Nuclear Environment of Disk Galaxies, vol. 439 of ASP Conf. Ser, 129 (2011).

16. Bar-Or, B. \& Alexander, T. Steady-state Relativistic Stellar Dynamics Around a Massive Black hole. Astrophys. J. 820, 129 (2016).

17. Magorrian, J. \& Tremaine, S. Rates of tidal disruption of stars by massive central black holes. Mon. Not. R. Astron. Soc. 309, 447-460 (1999).

18. Merritt, D. Dynamics and Evolution of Galactic Nuclei (Princeton: Princeton University Press, 2013).

19. Stone, N. C., Küpper, A. H. W. \& Ostriker, J. P. Formation of massive black holes in galactic nuclei: runaway tidal encounters. Mon. Not. R. Astron. Soc. 467, 4180-4199 (2017). 1606.01909.

20. Ayal, S., Livio, M. \& Piran, T. Tidal Disruption of a Solar-Type Star by a Supermassive Black Hole. Astrophys. J. 545, 772-780 (2000).

21. Bar-Or, B., Kupi, G. \& Alexander, T. Stellar Energy Relaxation around a Massive Black Hole. Astrophys. J. 764, 52 (2013).

22. Xiao, T. et al. Exploring the Low-mass End of the $\mathrm{M}_{B H}-\sigma_{*}$ Relation with Active Galaxies. Astrophys. J. 739, 28 (2011).

23. Salviander, S. \& Shields, G. A. The Black Hole Mass-Stellar Velocity Dispersion Relationship for Quasars in the Sloan Digital Sky Survey Data Release 7. Astrophys. J. 764, 80 (2013).

24. Sijacki, D. et al. The Illustris simulation: the evolving population of black holes across cosmic time. Mon. Not. R. Astron. Soc. 452, 575-596 (2015).

25. Taylor, P. \& Kobayashi, C. Time evolution of galaxy scaling relations in cosmological simulations. Mon. Not. R. Astron. Soc. 463, 2465-2479 (2016).

26. Chen, X., Sesana, A., Madau, P. \& Liu, F. K. Tidal Stellar Disruptions by Massive Black Hole Pairs. II. Decaying Binaries. Astrophys. J. 729, 13 (2011).

27. Walker, M. G. et al. A Universal Mass Profile for Dwarf Spheroidal Galaxies? Astrophys. J. 704, 1274-1287 (2009).

28. Baldassare, V. F., Reines, A. E., Gallo, E. \& Greene, J. E. A $\sim 50,000 M_{\odot}$ Solar Mass Black Hole in the Nucleus of RGG 118. Astrophys. J. 809, L14 (2015).

29. Kızıltan, B., Baumgardt, H. \& Loeb, A. An intermediate-mass black hole in the centre of the globular cluster 47 Tucanae. Nature 542, 203-205 (2017). 
30. Dubois, Y. et al. Black hole evolution - I. Supernova-regulated black hole growth. Mon. Not. R. Astron. Soc. 452, 1502-1518 (2015).

31. Fialkov, A. \& Loeb, A. Jetted Tidal Disruptions of Stars as a Flag of Intermediate Mass Black Holes at High Redshifts. ArXiv eprints (2016). 1611.01386

32. Rosswog, S., Ramirez-Ruiz, E. \& Hix, W. R. Tidal Disruption and Ignition of White Dwarfs by Moderately Massive Black Holes. Astrophys. J. 695, 404-419 (2009).

33. Stone, N. C. \& Metzger, B. D. Rates of stellar tidal disruption as probes of the supermassive black hole mass function. Mon. Not. R. Astron. Soc. 455, 859-883 (2016).

34. Yagi, K. Scientific Potential of Decigo Pathfinder and Testing GR with Space-Borne Gravitational Wave Interferometers. International Journal of Modern Physics D 22, 1341013 (2013).

35. Bahcall, J. N. \& Wolf, R. A. Star distribution around a massive black hole in a globular cluster. Astrophys. J. 209, 214-232 (1976).

36. Lightman, A. P. \& Shapiro, S. L. The distribution and consumption rate of stars around a massive, collapsed object. Astrophys. J. 211, 244-262 (1977).

37. Hoyle, F. \& Lyttleton, R. A. The effect of interstellar matter on climatic variation. Proceedings of the Cambridge Philosophical Society 35, 405 (1939)

38. Bondi, $\mathrm{H}$. On spherically symmetrical accretion. Mon. Not. R. Astron. Soc. 112, 195-+ (1952).

39. Alexander, T. \& Natarajan, P. Rapid growth of seed black holes in the early universe by supra-exponential accretion. Science $\mathbf{3 4 5}$ 1330-1333 (2014). 1408.1718

40. Murphy, B. W., Conn, H. N. \& Durisen, R. H. Dynamical and luminosity evolution of active galactic nuclei - Models with a mass spectrum. Astrophys. J. 370, 60-77 (1991).

41. Freitag, M. \& Benz, W. A new Monte Carlo code for star cluster simulations. II. Central black hole and stellar collisions. Astron. Astrophys. 394, 345-374 (2002).

42. Tremaine, S. et al. The Slope of the Black Hole Mass versus Velocity Dispersion Correlation. Astrophys. J. 574, 740-753 (2002).

43. Syer, D. \& Ulmer, A. Tidal disruption rates of stars in observed galaxies. Mon. Not. R. Astron. Soc. 306, 35-42 (1999). 